\title{
An Extended Non-Lane-Based Optimal Velocity Model with Dynamic Collaboration
}

\author{
Zhipeng Li and Run Zhang \\ The Key Laboratory of Embedded System and Service Computing of Ministry of Education, Tongji University, \\ Shanghai 201804, China
}

Correspondence should be addressed to Zhipeng Li; lizhipeng@tongji.edu.cn

Received 15 November 2012; Revised 1 February 2013; Accepted 1 February 2013

Academic Editor: Valentina E. Balas

Copyright (c) 2013 Z. Li and R. Zhang. This is an open access article distributed under the Creative Commons Attribution License, which permits unrestricted use, distribution, and reproduction in any medium, provided the original work is properly cited.

Incorporating the effects of the lane width in traffic, in this paper, we propose a dynamical model based on the strategy of threevehicle cooperation driving. We obtain the smoother acceleration distribution in the new model through considering the dynamic collaboration with the nearest preceding vehicle and the nearest following vehicle. It is proved that the stability of the new model is greatly improved compared to the early non-lane-based car following model by using the linear stability theory. We find that when the parameter of lateral separation distance is identified, the amplitude of traffic congestion decreases with increasing the strength of dynamic collaboration in the simulation experiments. In addition, we apply the new extended model to simulate the motions of cars starting from a traffic signal and the dissipating of the traffic congestion; it is found that our new model can predict realistic delay time and kinematic wave speed and obtained a faster dissipation speed of traffic congestion than the traffic flow model without considering the dynamic collaboration.

\section{Introduction}

Transportation has become one of the most concern issues in our daily life because the rapid development of modern society relies on the mobility of staff severely. In order to study the problems existing in the traffic, many mathematical models have been done in describing the dynamics of discrete groups of cars by an increasing number of investigators with different backgrounds and points of views. From the research perspective there are essentially two different types of approaches to studying traffic dynamics, namely, macroscopic and microscopic models. As one of the latter models, the car following model in traffic flow has been theoretically studied for nearly 50 years, from the earliest car following model proposed by Pipes in 1953 [1]. Its basic idea is that the current vehicle accelerates when it is slower than its leading vehicle, and decelerates as it is faster than its leading vehicle. This is the easiest and the most basic car following model. Later, in 1995 Bando et al. [2] proposed the optimal velocity model (OVM), which can successfully describe the dynamical formation of traffic congestion. Thereafter, there is a spring tide about the study of the car following model in traffic flow, based on optimal velocity model (OVM). For example, Helbing [3] and Treiber et al. [4] presented the generalized force model (GFM), in which high acceleration and unrealistic deceleration in simulating real traffic do not appear. In 2009, Gaididei et al. [5] conducted a detailed analysis of GFM and found out that although the GFM model has better agreement with the field data than original OVM, it cannot predict correctly the delay time of car motion and the kinematic wave speed at jam density; then they suggested a full velocity difference model (FVDM).

Based on the fact that real-time reporting systems of car information are now becoming widely available, in order to aid emergency dispatch assistance and traffic control management as being an important part of intelligent transportation system (ITS), the study has been focused on using the information of many other cars to suppress the appearance of traffic jams efficiently in traffic flow modeling. By taking the next nearest neighbor interaction into traffic flow modeling, a lattice model of optimized traffic flow with the consideration of optimal current was proposed by Xue [6]. Besides, taking the effect of information about the two leading vehicles to the following vehicle, Peng et al. [7] advised a two-car following 
model (TCFM) and Ge et al. [8] proposed a two-velocity difference model (TVDM). The difference in the two model is that the headway is one of the crucial parameters in the traffic flow between the second nearest neighbor vehicle and the following vehicle. Moreover, considering the effect of multivelocity difference ahead, an extended multi-look-ahead driving model is presented by Mo et al. [9]. Considering the multi-velocity difference ahead, it is indicated that the stability is improved greatly and the energy consumption of the traffic flow is reduced obviously. However, all of their work focuses on the information multivehicles in front [1012 . And it has been proved that the stability of traffic flow will be improved by taking preceding vehicles into consideration. The effect of the following vehicle is not taken into account until Gaididei et al. [5] and Nakayama et al. [13] raised an extended OV model, looking at the following vehicle, which can improve the stability of traffic flow as well $[14,15]$.

Apart from considering the factor of other vehicle's motion information, the effects of lane width have been studied to participate in the traffic flow modeling. In 2010 Hadiuzzaman and Rahman [16] pointed out the importance of the lane width on vehicle driving behaviors and Jin et al. [17] have been sparing no effort to focus on the effects of the lane width on car following model and conclude that lane width effects have great influence on the stability of traffic flow and the capacity of traffic flow. This is quite natural and very necessary in construction of traffic flow model because in actual traffic fields the influence on the real traffic states is uninterrupted; traffic flow modeling without considering this factor will not truly describe the real traffic flow evolution.

However, to our knowledge, the existing researches using the information of many other cars to suppress the appearance of traffic jams efficiently in traffic flow modeling mostly concentrate on the speeds and the sites information of other vehicles. On the other hand, traffic flow modeling is to help building the vehicular dynamical equation, which determines the power output of each vehicle running on road. So the introduction of the other vehicle's motion information into microscopic modeling of each vehicle will finally convert to the power fine-tuning of a single vehicle in traffic $[18,19]$. In fact, the process of slowing down of the traffic congestion of traffic flow is the result of power fine-tuning of individual vehicle in road network, which gives us the hint that direct adjusting of power output of each vehicle based on the other vehicle's power assist may be helpful to result in the stabilization of the traffic flow. So in this paper we build a new traffic flow model by considering the dynamic collaboration with the nearest preceding vehicle and the nearest following vehicle. In order to describe the real traffic characteristics, the extended model also takes the effects of the lane width on vehicle driving behavior into consideration. We will examine whether the new consideration can improve the stability of the traffic flow, and we will also check whether our new model can predict realistic delay time and kinematic wave speed and obtain a faster dissipation speed of traffic congestion than the traffic flow model without considering the dynamic collaboration.

This paper is organized as follows. Firstly, considering the dynamic collaboration, we propose the model based on the non-lane-based car following model. Secondly, by means of the liner stability theory, the model's stability condition is derived. Thirdly, numerical simulations are carried out about the traffic jams, and flux-density fundamental diagram is obtained. In addition, the research about delay times and dissipation speed of traffic congestion is discussed later. Finally, the obtained results are summarized.

\section{Model}

The optimal velocity model, which is one of the most classical car following models, is based on the assumption that the acceleration of the $n$th vehicle at time $t$ is determined by the difference between the actual velocity, $v_{n}(t)$, and an optimal velocity, $V\left(\Delta x_{n}(t)\right)$, which depends on the headway $\Delta x_{n}(t)$ to the car in the front:

$$
\frac{d v_{j}(t)}{d t}=a\left[V\left(\Delta x_{j}(t)\right)-v_{j}(t)\right],
$$

where $a$ is the sensitivity coefficient representing the driver's reaction time, applied to the difference between the optimal velocity and the current velocity. $\Delta x_{j}(t)=x_{j-1}(t)-x_{j}(t)$ is the headway between vehicle $j-1$ and vehicle $j$ at time $t$.

All the vehicles in the original OVM assumptions are supposed in a straight line exactly in a single lane with no passing. But in real traffic fields the vehicles are usually not in a straight line and appear to canine in most time in real traffic situations as shown in Figure 1. Hence the effects of the lane width are taken into account in the non-lane-based car following model to improve the linear stability just as considered in [11]. So we get the new acceleration

$$
\frac{d v_{j}(t)}{d t}=a\left[V\left(\Delta x_{j, j-1}(t), \Delta x_{j, j-2}(t)\right)\right]
$$

where $\Delta x_{j, j-1}(t)=x_{j-1}(t)-x_{j}(t)$ indicates the distance headway between the vehicle $j+1$ and the vehicle $j$ at the time $t ; \Delta x_{j, j-2}(t)=x_{j-2}(t)-x_{j}(t)$ is headway between the vehicle $j+2$ and the vehicle $j$ at the time $t$. The function $V\left[\Delta x_{j, j-1}(t), \Delta x_{j, j-2}(t)\right]$ can be represented as

$$
\begin{aligned}
V & {\left[\Delta x_{j, j-1}(t), \Delta x_{j, j-2}(t)\right] } \\
& =V\left[\left(1-p_{j}\right) \Delta x_{j, j-1}+p_{j} \Delta_{j, j-2}\right],
\end{aligned}
$$

where $p_{j}$ is the effect parameter of lateral separation distance, which is obtained by the equation $p_{j}=\mathrm{LS}_{j} / \mathrm{LS}_{\max }$, and $\mathrm{LS}_{j}$ is the lateral separation distance between vehicle $j$ and vehicle $j-1$. When the leading vehicle was not on the same lane, it would not have impact on the following vehicle at all by the lateral separation distance increasing to the maximal lateral separation distance $\mathrm{LS}_{\max }$. Generally, $\mathrm{LS}_{\max }$ can be set as the typical lane width $(3.6 \mathrm{~m})$.

Similarly, we can get the acceleration of vehicle $j-1$ and vehicle $j+1$, respectively, as follows:

$$
\begin{aligned}
& \frac{d v_{j-1}(t)}{d t}=a\left[V\left(\Delta x_{j-1, j-2}(t), \Delta x_{j-1, j-3}(t)\right)\right], \\
& \frac{d v_{j+1}(t)}{d t}=a\left[V\left(\Delta x_{j+1, j}(t), \Delta x_{j+1, j-1}(t)\right)\right] .
\end{aligned}
$$




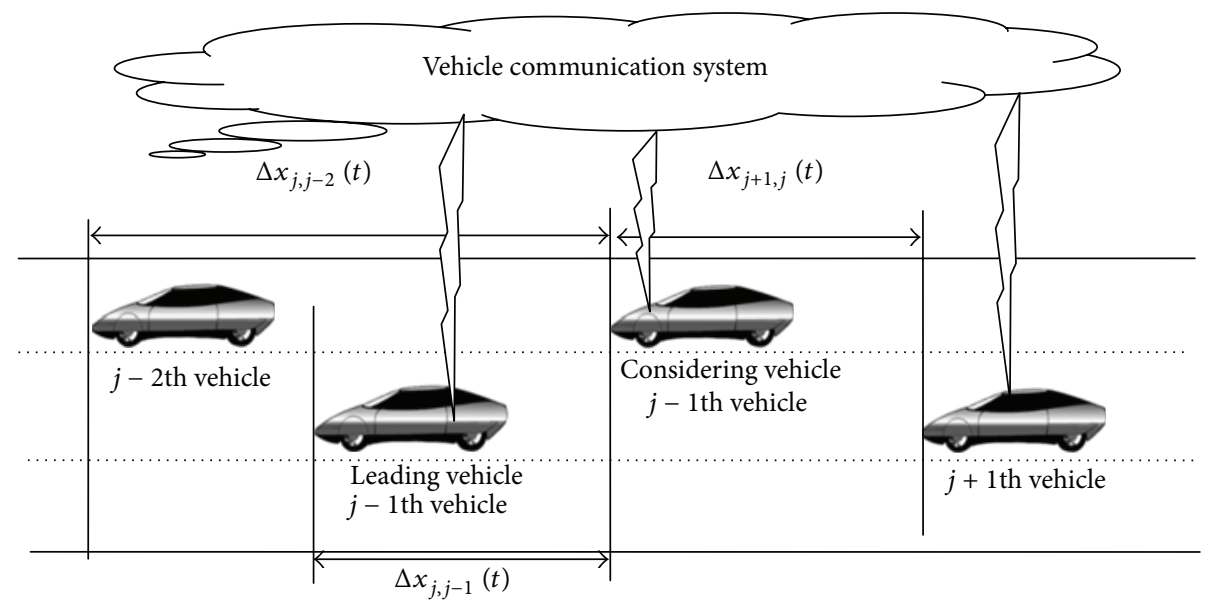

FIGURE 1: Schematic diagram for running vehicles.

Based on the discussion in Section 1, a new car following model is proposed here by extending the non-lane-based car following model to consider dynamic collaboration of the nearest preceding vehicle and the nearest following vehicle (shows in Figure 1). The acceleration of the considering vehicle and its nearest leading vehicle and following vehicle are obtained by the non-lane-based car following model. Besides, the movement of considering vehicle is influenced by its nearest leading vehicle and following vehicle greatly. Depending on both the acceleration of the nearest leading vehicle and that of the following vehicle, the acceleration of the considering vehicle is given as

$$
\begin{aligned}
\frac{d^{2} x_{j}(t)}{d t^{2}}= & k_{l}\left[V\left(\Delta x_{j-1, j-2}(t), \Delta x_{j-1, j-3}(t)\right)-x_{j-1}^{\prime}(t)\right] \\
& +k_{c}\left[V\left(\Delta x_{j, j-1}(t), \Delta x_{j, j-2}(t)\right)-x_{j}^{\prime}(t)\right] \\
& +k_{f}\left[V\left(\Delta x_{j+1, j}(t), \Delta x_{j+1, j-1}(t)\right)-x_{j+1}^{\prime}(t)\right],
\end{aligned}
$$

where the coefficients $k_{l}, k_{c}$, and $k_{f}$, are the sensitivity of vehicle $j+1, j, j-1$ separately. Generally, $k_{c}$ must be positive and (6) should be satisfied:

$$
k_{c}-\left(\left|k_{l}\right|+|k f|\right)>0
$$

Dynamic collaboration is adopted in our extended model, and its own acceleration must hold the dominant component, for it is obtained by the headway between its nearest leading and following vehicles, which have the greatest influence on the considering vehicle. So the weighting coefficients must hold (6).

From Figure 1, we find that every vehicle communicates with the vehicle communication system. Besides, each vehicle uploads its position and velocity to vehicle communication system and the acceleration is computed by (3) with the headway between its nearest leading and following vehicles. Then the acceleration is uploaded and the acceleration of the considering vehicle is adjusted by coupling the dynamics of its nearest leading and following vehicles. What is more, the weighting coefficients can be adjusted. Therefore, (5) is obtained. This is called dynamic collaboration.

Two important elements are considered in our proposed model (5). On one hand, the lateral space among the vehicles affects its moving states. On the other hand, dynamic collaboration has taken the information of the nearest leading vehicle and the nearest following vehicle into account with different weighting coefficients $k_{l}, k_{c}, k_{f}$.

There is a wide variety of optimal velocities to be selected in the model. Helbing and Tilch carried out a calibration of OVM with respect to the empirical data and suggested an optimal velocity function (OVF) as

$$
V\left(\Delta x_{j}(t)\right)=V_{1}+V_{2} \tanh \left[C_{1}\left(\Delta x_{j}(t)-l_{c}\right)-C_{2}\right],
$$

where $l_{c}=5 \mathrm{~m}$ is the length of vehicle. The other parameter values are determined as follows:

$$
\begin{aligned}
& V_{1}=6.75 \mathrm{~m} / \mathrm{s}, \quad V_{2}=7.91 \mathrm{~m} / \mathrm{s}, \\
& C_{1}=0.13, \quad C_{2}=1.57 .
\end{aligned}
$$

From our new extended model (5), we can observe that not only the lane width effects in car following model are incorporated to describe the real traffic fields, but also the dynamic collaborations of the nearest leading vehicle and the nearest following vehicle are used to smooth the acceleration. With the smoother acceleration, the stability of the traffic flow is expected to be further improved and the traffic jams at the high density will be efficiently suppressed by the new consideration.

\section{Linear Stability Analysis}

Next we explore whether the proposed model can further stabilize traffic flow with the new consideration of dynamic collaborations of the nearest preceding vehicle and the nearest following vehicle. We apply the linear stability method to the extended model described by (5). Besides, the linear stability method is adopted to estimate the other car following models, 
such as OVM and GHR. The same method and derivation process are applied in this section. The homogeneous traffic flow is defined by such a state that all vehicles run with the same headway $h$, the same optimal velocity $V(h, 2 h)$, and the same lateral separation $\mathrm{LS}_{j}$ to ensure the same $p_{j}$. Hence the steady state solution is given as

$$
x_{j}^{(0)}(t)=V(h, 2 h) t+j h, \quad h=\frac{L}{N},
$$

where $N$ is the total number of cars, $L$ is the length of the road, and $h$ is the steady headway. What is more, in the uniform traffic flow the optimal velocity can be obtained by the equation below

$$
V(h, 2 h)=V\left[\left(1-p_{j}\right) h+p_{j} 2 h\right],
$$

and suppose $y_{j}(t)$ to be a small deviation from the steady state solution $x_{j}^{0}(t)$, so

$$
x_{j}(t)=x_{j}^{(0)}(t)+y_{j}(t) .
$$

Substituting (11) and (9) into (5), it is turned into

$$
\begin{aligned}
y_{j}^{\prime \prime}(t)=k_{l}\left[V^{\prime}(h, 2 h)(\right. & \left(1-p_{j-1}\right) \Delta y_{j-1, j-2}(t) \\
& \left.\left.+p_{j-1} \Delta y_{j-1, j-3}(t)\right)-y_{j-1}^{\prime}(t)\right] \\
+ & k_{c}\left[V ^ { \prime } ( h , 2 h ) \left(\left(1-p_{j}\right) \Delta y_{j, j-1}(t)\right.\right. \\
& \left.\left.+p_{j} \Delta y_{j, j-2}(t)\right)-y_{j}^{\prime}(t)\right] \\
+k_{f}\left[V^{\prime}(h, 2 h)(\right. & \left(1-p_{j+1}\right) \Delta y_{j+1, j}(t) \\
& \left.\left.+p_{j+1} \Delta y_{j+1, j-1}(t)\right)-y_{j+1}^{\prime}(t)\right],
\end{aligned}
$$

where $\Delta y_{j, j-1}(t)=y_{j-1}(t)-y_{j}(t)$ and $V^{\prime}(h, 2 h)=d V\left(\Delta x_{j}\right) /$ $d \Delta x_{j} \mid \Delta x_{j}=\left(1+p_{j}\right) h$.

The $y_{j}(t)$ can be supposed in the Fourier models, that is, $y_{j}(t)=A \exp (i k j+z t)$ as it is a small deviation. Substituting it into (12), we obtain

$$
\begin{aligned}
z^{2}= & k_{c}\left[V^{\prime}(h)(\exp (i k)-1)-z\right] \\
& +k_{l}\left[V^{\prime}(h)(\exp (2 i k)-\exp (i k))-z \exp (i k)\right] \\
& +k_{f}\left[V^{\prime}(h)(1-\exp (-i k))-z \exp (-i k)\right] .
\end{aligned}
$$

Expanding $z=z_{1}(i k)+z_{2}(i k)^{2}+\cdots$ and substituting it into (13), the first- and second-order terms of coefficients in the expression of $z$ are given as

$$
\begin{aligned}
z_{1}= & V^{\prime}(h, 2 h)\left(1+p_{j}\right), \\
z_{2}= & \frac{V^{\prime}(h, 2 h)\left(1+3 p_{j}\right)}{2} \\
& -\frac{\left[V^{\prime}(h, 2 h)\right]^{2}\left(1+p_{j}\right)^{2}}{k_{l}+k_{c}+k_{f}} .
\end{aligned}
$$

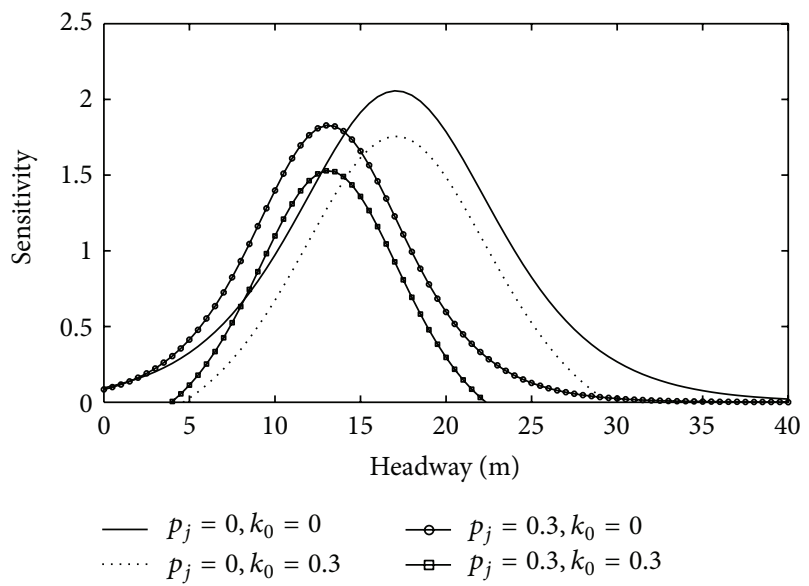

FIGURE 2: Neutral stability lines in the headway-sensitivity space with different $\left(p_{j}, k_{0}\right)$.

When $z_{2}>0$, the traffic flow is stable; then the neutral stability is obtained by

$$
a=k_{c}=\frac{2 V^{\prime}(h, 2 h)\left(1+p_{j}\right)^{2}}{1+3 p_{j}}-k_{l}-k_{f} .
$$

If $a<2 V^{\prime}(h, 2 h)\left(1+p_{j}\right)^{2} /\left(1+3 p_{j}\right)-k_{l}-k_{f}$, the traffic flow is unstable in the conditions, for small disturbances with long wavelengths.

To our knowledge, the proof method is widely used in the analysis of car following system and the derivation process is reasonable and concise. Finally, the method is applicative in our extended model.

Figure 2 shows that the neutral stability lines in the headway-sensitivity space with different $p_{j}$ and $k_{0}\left(k_{0}=\right.$ $k_{l}+k_{f}$ ), which are the circumscription between stable region and unstable region of the traffic flow. In the stable region, the traffic flow is stable and no jams occur. In addition, $k_{0}=$ $k_{l}+k_{f}$ is set to study the influence of the proposed model by the dynamic collaboration of both the nearest leading vehicle and the following vehicle on the traffic flow. Furthermore, it is obtained that the parameters $k_{l}$ and $k_{f}$ have the same influence on the stability from (15). However, the traffic flow is unstable below the neutral stability lines and a traffic jam will appear. From Figure 2, we can also find that the stability regions are enlarged with the increasing of $p_{j}$, which had been proved by Jin et al. [17]. Besides, the more we consider the dynamic collaboration of the nearest leading vehicle and the nearest following vehicle, the critical points and curves are more lowered in our new model based on the non-lane-based car following model. This means that when the parameter $p_{j}$ is fixed to a certain value, we also can enlarge the stable region with increasing the weight of dynamic collaboration by the augmentation of the parameter $k_{0}$. Apparently, the greater values of parameter $k_{0}$ can bring about the greater stability region. Then the simulation results will be listed in the next section. 


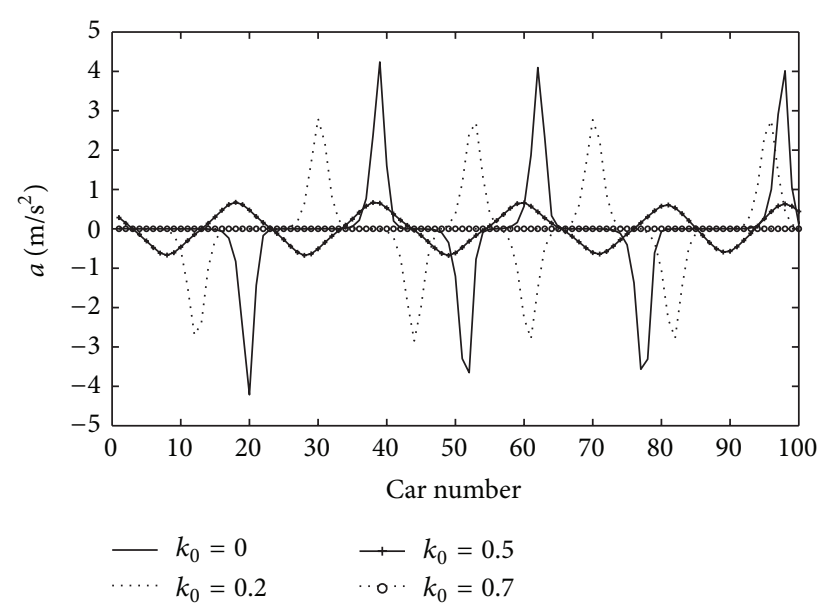

(a)

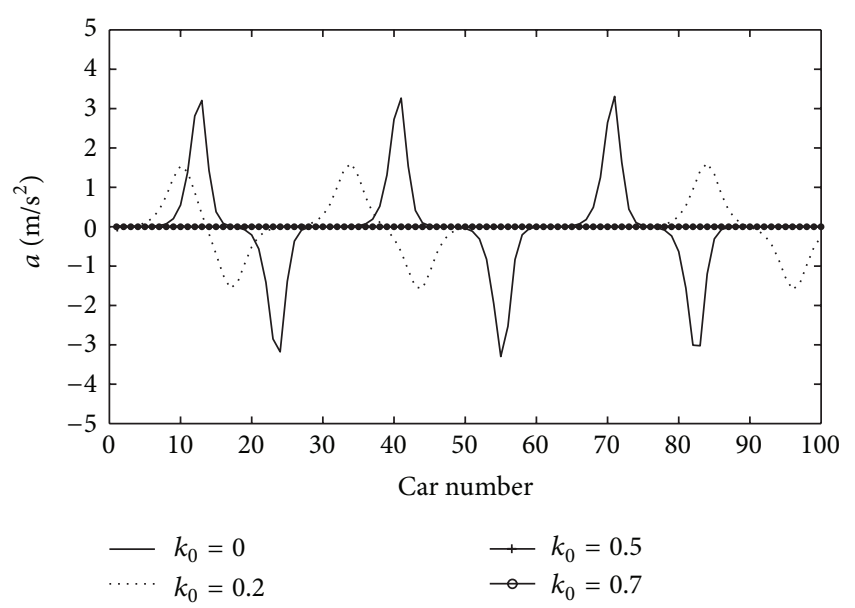

(b)

FIGURE 3: Acceleration profiles at sufficiently large time $t=10000 \mathrm{~s}$ for (a) $p_{j}=0.0$ and (b) $p_{j}=0.1$.

\section{Results}

To check the validity of our analysis above, we apply (5) to study numerical simulation for our model described by (5) under the periodic boundary condition. At the beginning, there are 100 vehicles moving with the same optimal velocity $V(h, 2 h)$ on a ring road of $1700 \mathrm{~m}$. According to empirical data, we set the value of the parameter $k_{c}, k_{c}=a=1.5$. What is more, the initial simulation conditions and small perturbations are given as

$$
\begin{aligned}
& \Delta x_{1}(0)=18 \mathrm{~m}, \quad \Delta x_{2}(0)=16 \mathrm{~m}, \\
& \Delta x_{j}(0)=17 \mathrm{~m} \quad(j \neq 1,2), \\
& x_{j}^{\prime}(0)=V(h, 2 h) .
\end{aligned}
$$

Figure 3 shows the acceleration profiles at sufficiently large time $t=10000 \mathrm{~s}$ with the different parameters $p_{j}$ and $k_{0}$. From this figure, we can observe that the accelerations of these vehicles are smoother with the increasing of the $p_{j}$ with the same value of $k_{0}$, which shows that the lateral separation effects can strengthen the stability of the uniform traffic flow and the traffic jams are suppressed efficiently as proved by Jin [17]. Moreover, if we fixed the lateral separation effects with $p_{j}=0.1$, we can obtain the typical spatiotemporal evolution of the headway after sufficiently long time $t=10^{4} \mathrm{~s}$ for different value of the $k_{0}$, which is the weight of dynamic collaboration of the nearest leading vehicle and the nearest following vehicle as shown in Figure 4. We can see that the phase transit from free flow to jammed traffic is observed and the jams propagate backward as the kink-antikink density waves. In Figure 4(a), our new model will turn to the nonlane-based optimal velocity car following model as $k_{l}=k_{f}=$ 0 is set. Figure 5 shows the headway profile obtained at sufficiently large time $t=10^{4} \mathrm{~s}$. With increasing $k_{0}$, and the weight of dynamic collaboration of the nearest leading vehicle and the nearest following vehicle, the amplitude of the density wave is weakened, initial small disturbances decay, and initial traffic flow with a nonuniform density profile evolves to a uniformly traffic flow which is shown in pattern (b) of Figure 3 if we set $p_{j}=0.1$ and $k_{0}=0.13$. Compared to the model without dynamic collaboration, all the results show that the new model considering the dynamic collaboration of the nearest leading vehicle and the nearest following vehicle can improve the stability of the traffic flow.

Figure 6 shows the flux density diagram plots of the traffic flow for different value of $k_{0}$. Just as we see from the picture, applying the smooth acceleration obtained by dynamic collaboration, we can receive the glossy curve of flux density in the new model. The three states, divided by the neutral stability lines, are free flow (state I), synchronized flow (state II), and homogeneous traffic flow (state III). Besides, vehicles move freely with low density in state I, and the traffic flux mutates with the density among a certain reign. With the higher density, the traffic flow enters into homogeneous flow. However, the traffic flux mutates when the density is higher than the large critical value and is lower than the little critical value in other car following models. Clearly, there is no traffic flux mutation in our extended model, that is to say, our traffic flow is stable and the new model can suppress the mutation with increasing the $k_{0}$. Besides, the congestion dissipation is discussed in Section 5.

\section{Delay Times and Dissipating Speed of the Traffic Congestion}

The two parameters, delay times and dissipating speed, are important to measure car following model. However, lateral space and dynamic collaboration with its nearest leading and following vehicles are considered in our extended model, which are not hoped to worsen the two important parameters. In this section, we apply the new extended model to simulate the motions of cars starting from a traffic signal to find out the difference between our extended model and other classical models like FVD about the delay times and the kinematic wave speed. If velocities of two successive cars are given by 


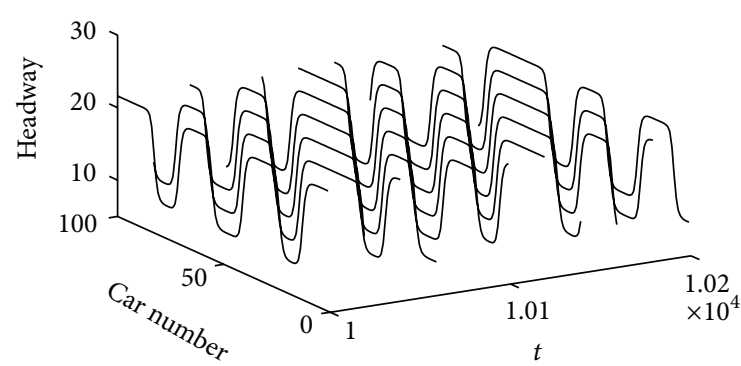

(a) $p_{j}=0.1, k_{0}=0.0$

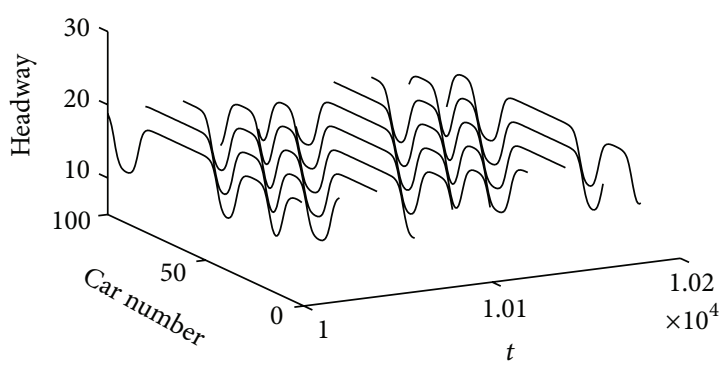

(c) $p_{j}=0.1, k_{0}=0.125$

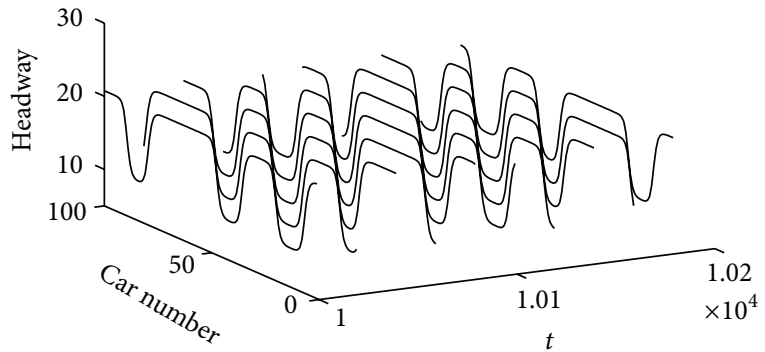

(b) $p_{j}=0.1, k_{0}=0.1$

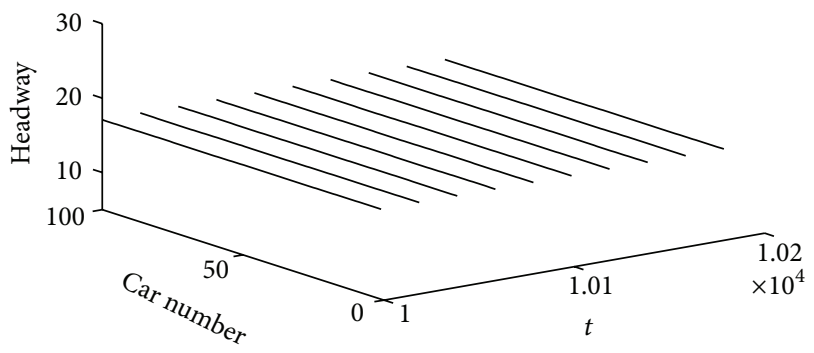

(d) $p_{j}=0.1, k_{0}=0.13$

Figure 4: Space-time evolution of the headway after 1000000 time steps for different $p_{j}$ and $k_{0}$.

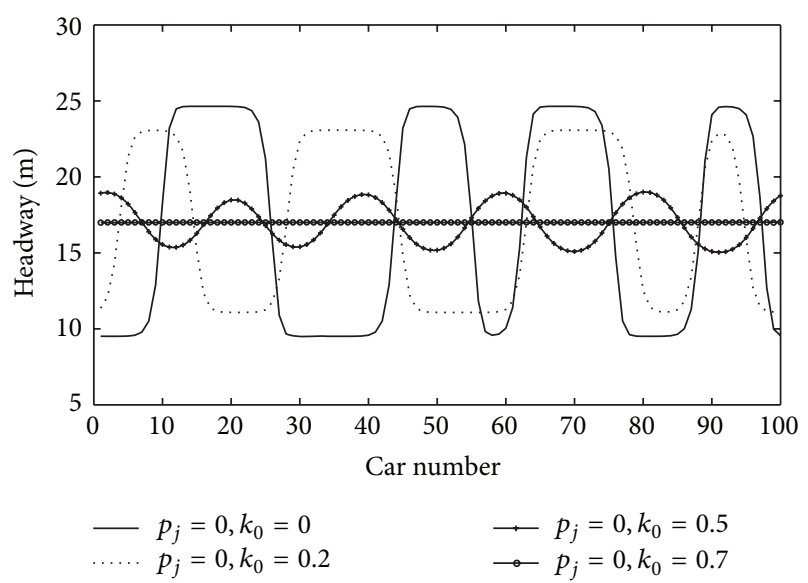

(a)

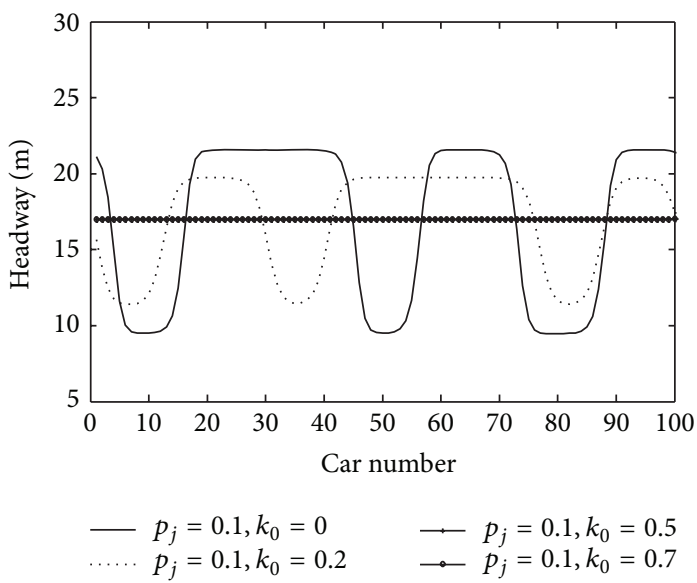

(b)

FIgURE 5: A snapshot of headway of all cars at $t=10,000 \mathrm{~s}$ for (a) $p_{j}=0.0$ and (b) $p_{j}=0.1$.

$v(t)$ and $v(t-T)$, respectively, the delay time of car motion is $T$ and the kinematic wave speed $v_{k}$ is equal to the quotient of the headway $7.4 \mathrm{~m}$ divided by $T$. The delay time of car motion is about one second in actual observation pointed out by Bando. Besides, Del Castillo and Benitez put forward that the range of disturbance propagation speed of car motions is from $17 \mathrm{~km} / \mathrm{s}$ to $23 \mathrm{~km} / \mathrm{s}$. We assume that the traffic signal is red and all cars are waiting with a headway of $7.4 \mathrm{~m}$ except the first car for the optimal velocity is zero with the headway obtained by (7), that there are 20 vehicles waiting at the traffic signal with the identical headway $h$, and that all cars start and pass through the bottleneck region one by one at time $t=0$.
Figure 7 shows the results about delay times and disturbance propagation speed in the simulation of cars motion, from which the delay time is obtained with the two different parameters $k_{l}$ and $k_{f}$ in patterns (a) and (b), respectively. The new extended model degenerates to that without the effect of the dynamic collaboration of the nearest leading vehicle and the nearest following vehicle at parameters $k_{l}=k_{f}=$ 0.0 in pattern (a); that is to say, the current vehicle moves freely without constraints of dynamic collaboration with the nearest forward and nearest backward vehicles. Compared to pattern (a), the delay time becomes shorter and disturbance propagation speed is still in the region which was pointed out 


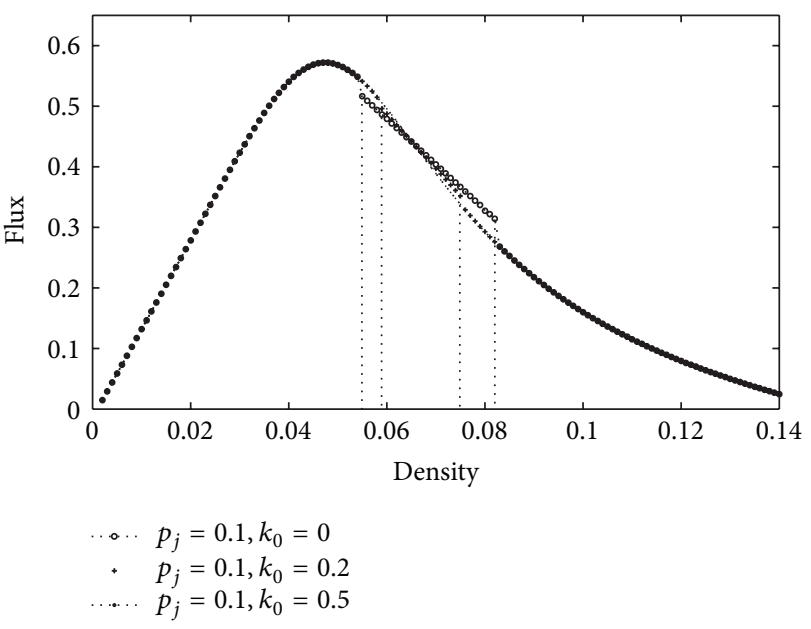

Figure 6: Flux-density profiles plot for different $k_{0}$.

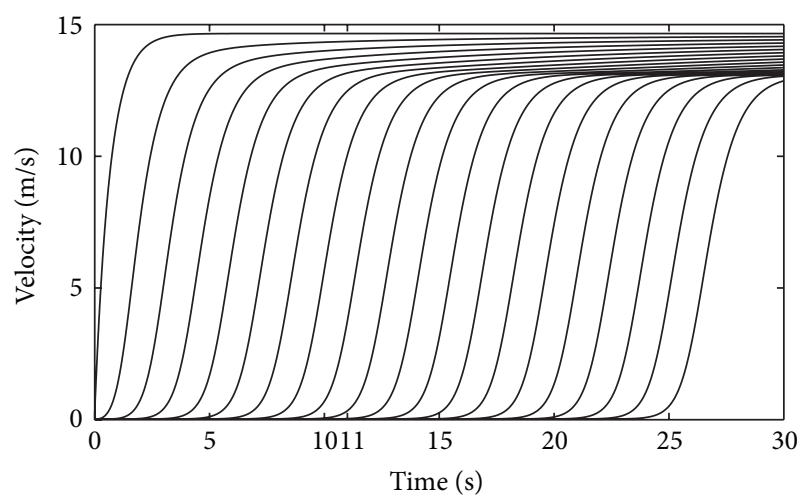

(a) $k_{l}=k_{f}=0.0$

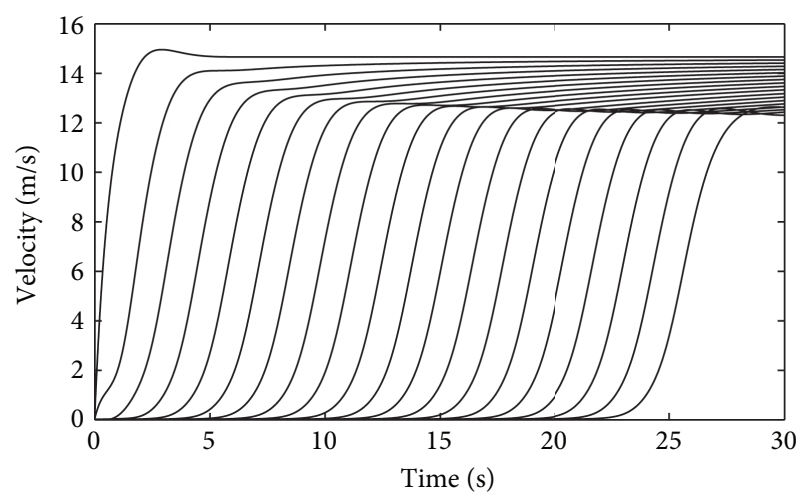

(b) $k_{l}=k_{f}=0.3$

FIGURE 7: Motions of cars starting from a traffic signal.

by Del Castillo and Benitez with dynamic collaboration by the new extended model in pattern (b), in which we set the parameters $k_{l}=k_{f}=0.3$.

The difference between the model without the dynamic collaboration and the new extended model with the dynamic collaboration in delay times and disturbance propagation speed is revealed in Table 1 intuitively. The longer delay time with the dynamic collaboration is not hoped to appear. For
TABLE 1: Delay times and disturbance propagation speed of car motion from a traffic in different model.

\begin{tabular}{lcc}
\hline Model & $\delta t(\mathrm{~s})$ & $C_{j}(\mathrm{~km} / \mathrm{h})$ \\
\hline Without the dynamic collaboration & 1.38 & 19.30 \\
With the dynamic collaboration & 1.32 & 20.18 \\
\hline
\end{tabular}

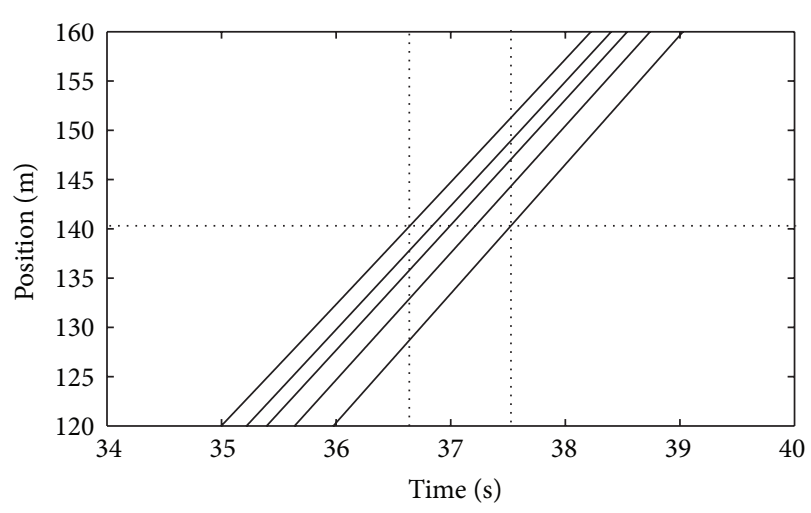

Figure 8: Congestion dissipation for different $k_{0}$.

comparison, we use the same sensitivity coefficient as that in the new model. From Table 1, we can see that the both models are in accord with the theoretical value, and the delay time is improved a little even more. Therefore, the new extended model is successful in anticipating the delay times and disturbance propagation speed with the shorter delay times.

What's more, our extended model is useful to dissipating the traffic congestion which is proved in the simulation from Figure 8 as the traffic congestion is becoming more and more serious, especially in Beijing, Shanghai, and other large cities with more vehicles and prosperous economy. In the simulation, we assume that a sufficient number of vehicles wait in the queue in a traffic bottleneck at the beginning and start to accelerate with the smooth acceleration in dynamic collaboration one by one at the time $t>0$. Here, we take the point that the departure of vehicles from a traffic light and the dissipation from a traffic jam have similar dissipation process. We keep an account of the leaving time by the 20th vehicle and compare the total time of congestion dissipation for the new model with and without the dynamical collaboration.

From Figure 8, it is found that the traffic congestion dissipates faster with increasing the weight of dynamic collaboration. The line of the rightmost represents the parameters $k_{l}=$ $k_{f}=0.0$ with no consideration of dynamic collaboration and the leftmost line stands for the displacement evolution of the twentieth vehicle at the parameters $k_{l}=k_{f}=0.5$. Apparently, the congestion dissipation is improved successfully by the consideration of dynamic collaboration.

\section{Conclusion}

Incorporating the effects of the lane width in traffic, in this paper, we propose a new model based on the strategy of threevehicle cooperation driving. Through theoretical analysis and simulations, it has been verified that by introducing 
the dynamic collaboration of the nearest preceding vehicle and the nearest following vehicle and the effects of the lane width in traffic, the extended model can further improve the stability of traffic flow. Through analyzing the evolution of stop-and-go waves, it has been observed that with the same effect of the line width, traffic congestions can be suppressed efficiently by increasing the strength of dynamic collaboration of the nearest preceding vehicle and the nearest following vehicle. Through studying traffic states and jamming transitions, the improvement of stability of the traffic flow has been further verified along with the increasing intensity of the dynamic collaboration and effect of lane width. In addition, the new extended model is arranged to simulate the motions of cars starting from a traffic signal and the dissipating of the traffic congestion; it is concluded that our new model with the dynamic collaboration can predict realistic delay time and kinematic wave speed, and obtain a faster dissipation speed of traffic congestion than the traffic flow model without considering the dynamic collaboration. In future, the research about the traffic characteristics influenced by the none-lane-change model and the different models taken into the lane changing condition will be our direction.

\section{Acknowledgments}

This work is supported by the Natural Science Foundation of China under Grant no. 60904068, Natural Science Foundation of China under Grant no. 10902076, Natural Science Foundation of China under Grant no. 11072117, Natural Science Foundation of China under Grant no. 61004113, the Fundamental Research Funds for the Central Universities under Grant no. 0800219198 and Natural Science Foundation of Shanghai under Grant no. 12ZR1433900.

\section{References}

[1] L. A. Pipes, "An operational analysis of traffic dynamics," Journal of Applied Physics, vol. 24, no. 3, pp. 274-281, 1953.

[2] M. Bando, H. Hasebe, and A. Nakayama, "Dynamical model of traffic congestion and numerical-simulation," Physical Review E, vol. 51, no. 2, pp. 1035-1042, 1995.

[3] D. Helbing, "Generalized force model of traffic dynamics," Physical Review E, vol. 58, no. 1, pp. 133-138, 1998.

[4] M. Treiber, A. Hennecke, and D. Helbing, "Derivation, properties, and simulation of a gas-kinetic-based, nonlocal traffic model," Physical Review E, vol. 59, no. 1, pp. 239-253, 2003.

[5] Y. B. Gaididei, R. Berkemer, and J. G. Caputo, "A Analytical solutions of jam pattern formation on a ring for a class of optimal velocity traffic models," New Journal of Physics, vol. 11, 2009.

[6] Y. Xue, "A car-following model with stochastically considering the relative velocity in a traffic flow," Acta Physica Sinica, vol. 52, no. 11, pp. 2750-2756, 2003.

[7] G. H. Peng, D. H. Sun, and H. P. He, "Two-car following model of traffic flow and numerical simulation," Acta Physica Sinica, vol. 57, no. 12, pp. 7541-7546, 2008.

[8] H. X. Ge, R. J. Cheng, and Z. P. Li, "Two velocity difference model for a car following theory," Physica A, vol. 387, pp. 52395245, 2008.
[9] Y. L. Mo, H. D. He, and Y. Xue, "Effect of multi-velocity-difference in traffic flow," Chinese Physics B, vol. 17, no. 12, pp. 44464450, 2008.

[10] H. Ge, Y. Liu, and R. Cheng, "A modified coupled map car following model and its traffic congestion analysis," Communications in Nonlinear Science and Numerical Simulation, vol. 17, no. 11, pp. 4439-4445, 2012.

[11] R. Manzo, B. Piccoli, and L. Rarità, "Optimal distribution of traffic flows in emergency cases," European Journal of Applied Mathematics, vol. 23, no. 4, pp. 515-535, 2012.

[12] N. Sugiyama and T. Nagatani, "Multiple-vehicle collision induced by a sudden stop in traffic flow," Physics Letters A, vol. 376, no. 22, pp. 1806-1806, 1803.

[13] A. Nakayama, Y. Sugiyama, and K. Hasebe, "Soliton solutions of exactly solvable dissipative systems," Computer Physics Communications, vol. 142, no. 1-3, pp. 259-262, 2001.

[14] S. Jin, D. Wang, and C. Xu, "Staggered car-following induced by lateral separation effects in traffic flow," Physics Letters A, vol. 376, no. 3, pp. 153-157, 2012.

[15] J. Tian, B. Jia, and X. Li, "A new car following model: comprehensive optimal velocity model," Communications in Theoretical Physics, vol. 55, no. 6, pp. 1119-1126, 2011.

[16] M. Hadiuzzaman and M. M. Rahman, "Capacity analysis for fixed-time signalized intersection for non-lane based traffic condition," Advances in Materials and Processing, vol. 83, pp. 904-913, 2010.

[17] S. Jin, D. Wang, and P. Tao, "Non-lane-based full velocity difference car following mode," Physica A, vol. 389, pp. 4654-4662, 2010.

[18] M. Batista and E. Twrdy, "Optimal velocity functions for car-following models," Journal of Zhenjiang University Science A, vol. 11, no. 7, pp. 520-529, 2010.

[19] N. Jia and S. Ma, "Comparison between the optimal velocity model and the Nagel-Schreckenberg model," Acta Physical Sinica, vol. 59, no. 2, pp. 832-841, 2010. 


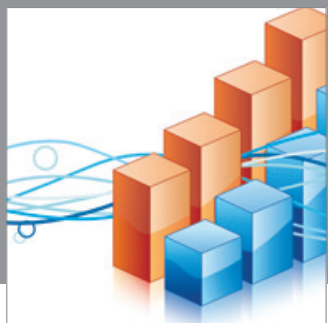

Advances in

Operations Research

mansans

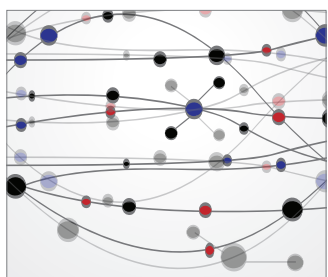

The Scientific World Journal
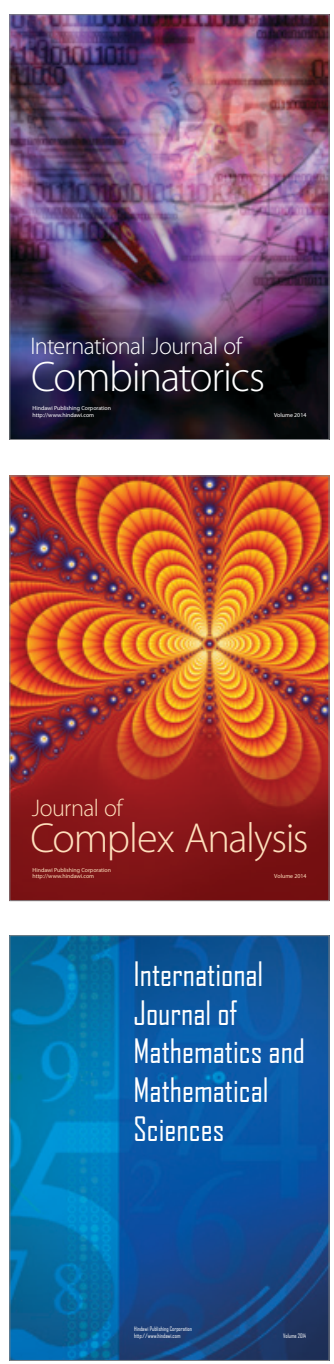
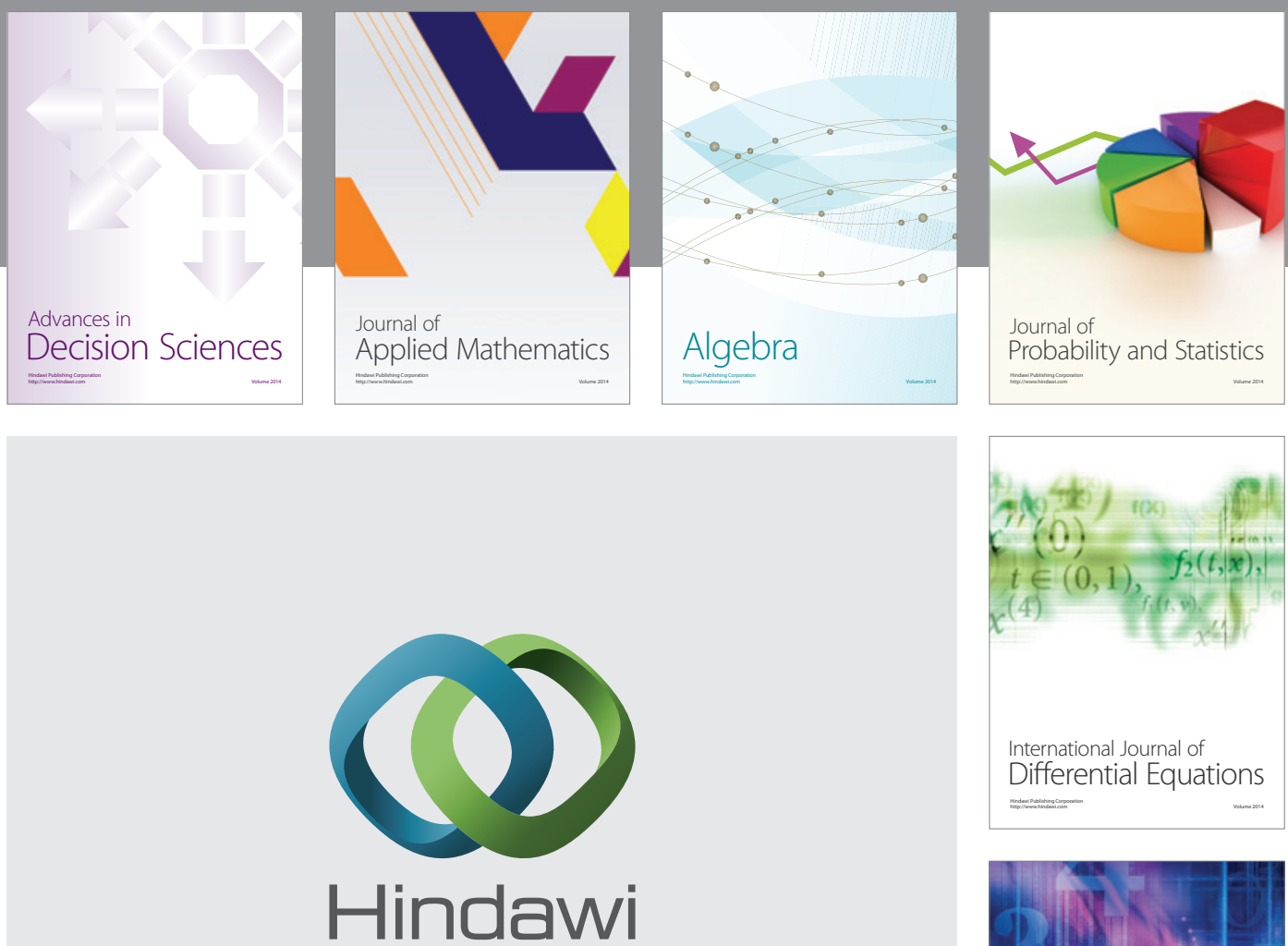

Submit your manuscripts at http://www.hindawi.com
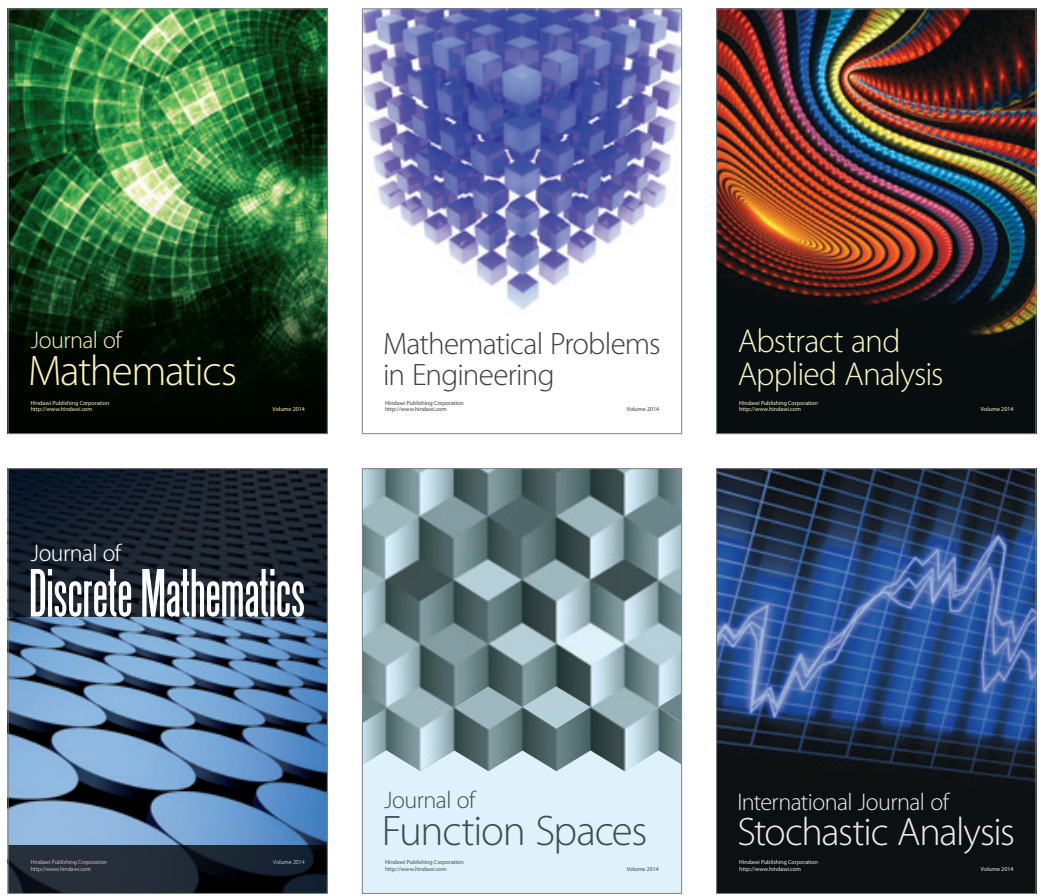

Journal of

Function Spaces

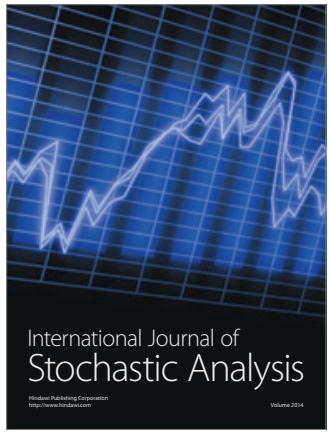

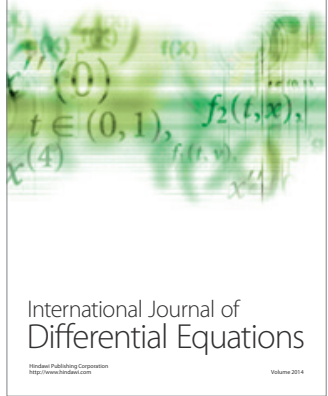
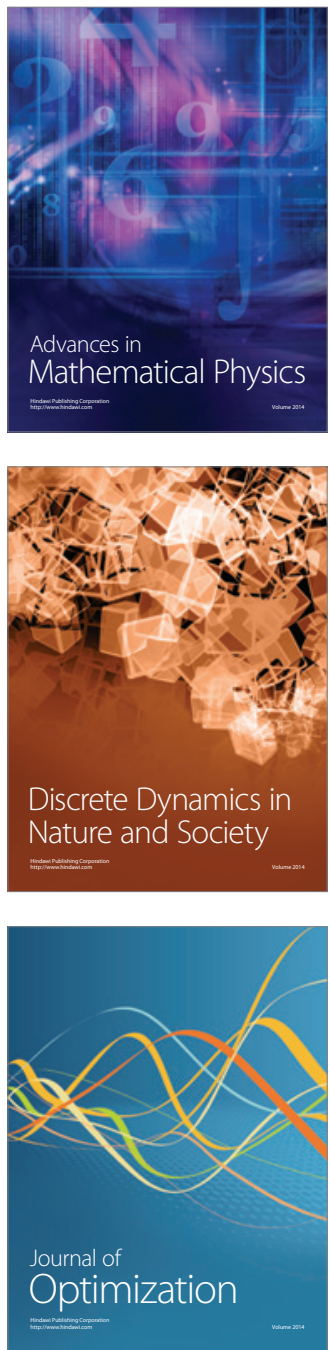